\title{
The Technology of Differentiated Instruction in Text Programming in Elementary School Based on the Website dl.gsu.by
}

\author{
Michael DOLINSKY, Mariya DOLINSKAYA \\ Faculty of Mathematics and Technologies of Programming, F. Skorina \\ Gomel State University, Sovetskaya str., 104, Gomel. 246019. Republic of Belarus \\ e-mail:dolinsky@gsu.by,mkugejko@gsu.by
}

\begin{abstract}
The technology of teaching text-based programming on the basis of the website DL.GSU.BY is described. The main advantages of the technology include: "zero entry threshold", training adapted to the pupil, many years of practical experience, effectiveness and scalability.
\end{abstract}

Keywords: distance learning, textual programming, primary school, Olympiad in Informatics, website DL.GSU.BY, F. Skorina Gomel State University.

\section{Introduction}

The authors have been actively preparing schoolchildren and students for Olympiads in Informatics and programming for many years (Dolinsky, 2016). Since 1997, this work has been carried out on the basis of the computer science cabinet of secondary school No. 27 in Gomel. Since 1999, this work has been actively supported by the distance learning website DL.GSU.BY of F. Skorina Gomel State University. An important distinguishing feature of the authors' approach is the built-in text-based training system in elementary school, the description of which this work is devoted to, emphasizing the following advantages of the authors' approach:

- Zero entry thresholds.

- Propaedeutics of text programming.

- Developing interesting differentiated training.

- Task - oriented training.

- Minimalist approach to theory.

- Regional programming Olympiads for pupils in grades 1-4.

- Preparation for the Olympiads of grades 5-8 (general tasks with the Olympiads of grades 5-8). 
- Problems in school mathematics, Olympiad mathematics and informatics mathematics.

- Competitions motivating to permanent studies.

- Many years of practical experience.

- Experience-based training scalability.

- Productivity.

- Low requirements for teacher professional qualifications.

- "Accelerated Course 2013".

- Support for the transition to the study of $\mathrm{C}++$.

\section{Zero Entry Thresholds}

A programming training system based on the DL.GSU.BY website was conceived as a means of helping a teacher teaching in a computer class. But since in the learning process we permanently reduced the age at which classes began (grade 10, grade 8, grade 5, grade 1), it became fundamentally important to ensure selfeducation, because otherwise the effectiveness of joint learning of a group of children would drop sharply. Moreover, in the secondary school No. 27 of the city of Gomel, the work is done frontally - that is, with all elementary school pupils whose parents stated in writing that they want informatics classes to be held with their children. As a rule, almost $100 \%$ of parents write such statements.

In other words, in the secondary school No. 27 of the city of Gomel we teach all pupils, not selected ones. Children who come to the first grade of our school are differently prepared for learning. This forced us to develop the educational system in such a way that not only well-prepared children, but also everyone else, would participate in our classes with interest and learn effectively. All first graders begin their studies with the course "Informatics 2015" (2015 means that it was formed and fixed in 2015) in the package of tasks "Learning to think 2012" (Dolinsky, 2014). This package is aimed at developing thinking skills, which helps to learn more productively in the future. The tasks of this package with five levels of difficulty develop the following basic mental operations:

- Operations on pairs: comparison, ordering, association.

- Operations on sets: union, intersection, subtraction.

- Operations on the set: classification, structuring, generalization.

- Logical operations: negation, conjunction, disjunction, equivalence, implication.

- Complex operations: synthesis, memorization, analysis, imagination, analogy, abstraction, positioning.

All tasks are focused on the ability to be performed by children who cannot read, for which they are presented in the form of a picture, some of the components of which need to be moved to another location with the mouse or just click on them. 
If the pupil cannot do the task, there is a button "I don't know", which transfers him to the tree of facilitating tasks. You can return back by completing the assignments, or by clicking the "I understand" button. Note that the "Learning to Think 2012" task package contains 620 main tasks and 1408 along with facilitating tasks.

In addition, for children who have systematic problems with some kind of mental operation, there are special task packages "Technical minimum. Differences", "Technical minimum. Analogy", etc., which are designed to improve the performing skills of such tasks from level zero to the ability to complete tasks in the main course "Learning to think".

For children who have systematic problems with all tasks, there is a special introductory package of tasks "Technical minimum. Learning to think - 0 ".

For children who do not own a mouse, there is a special package of tasks "Technical minimum. Learning to work with the mouse".

Finally, for children who needed a lot of help with the Learning to Think 2012 course, we created the Learning to Think (Quickly) course, which consists of 128 key tasks of the "Learning to Think" course (544 tasks with facilitators). Repetition is the mother of learning!

Thus, we have ensured that every first-grader of the secondary school No. 27 goes to computer science classes with pleasure, and at the same time, everyone is moving towards the development of basic mental skills to the level that provides effective follow-up training. Actually, in our system, children who have learned to walk and talk, that is, from 3-4 years old, can be engaged. And practice has shown that such classes can be conducted at home alone or with minimal help from parents.

\section{Propaedeutics of Text Programming}

Historically, text programming classes start with Pascal. The first barriers that must be overcome at the beginning of training are:

- Remember the order of keywords in the program (program, var, longint, begin, readln, writeln, end).

- Remember their translations into Russian.

- Be able to name an analogue of the Russian word in English and vice versa.

- Remember the spelling of each of the English words (in small and capital letters).

- Remember the location of the English letters of the studied keywords on the keyboard.

- Formulate sustainable skills for quick typing of keywords.

All these problems are solved within the framework of two packages of tasks "Propaedeutics of words" (207 main tasks, 787 with leading tasks) and "Learning words (slowly)" (127 tasks, 1491 with leading tasks) (Dolinsky and Dolinskaya, 2018). At the same time, the course "Propaedeutics of words" presents tasks that do not require memorization. Each task has the necessary hints up to the location of the letter on the keyboard. 
But the course "Learning words (slowly)" gradually helps the pupil to remember the order of letters in each word, their location on the keyboard, to train their typing. Moreover, the study of a new word ends with a control set of all learned words together.

As a result, almost all children cope with the task, although, of course, they do this with a significant difference in the time spent.

The propaedeutics of text programming is completed by studying the course "Number" (Dolinsky and Dolinskaya, 2019) containing 398 main tasks, along with 1244 auxiliary tasks. It begins with a slow transition from keywords to the first program (enter and display a number), presented below:

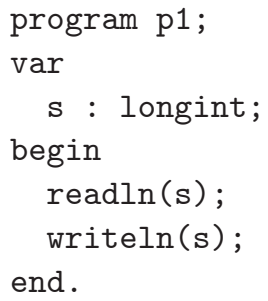

And the ultimate goal of training in the "Number" course is to teach pupils to solve the first three problems of the 20 problems of the Olympiad in programming for pupils in grades 1-4 for input, formatted output, and simple number processing. The conditions for such tasks at the regional Olympiad held on April 20, 2018 are given below:

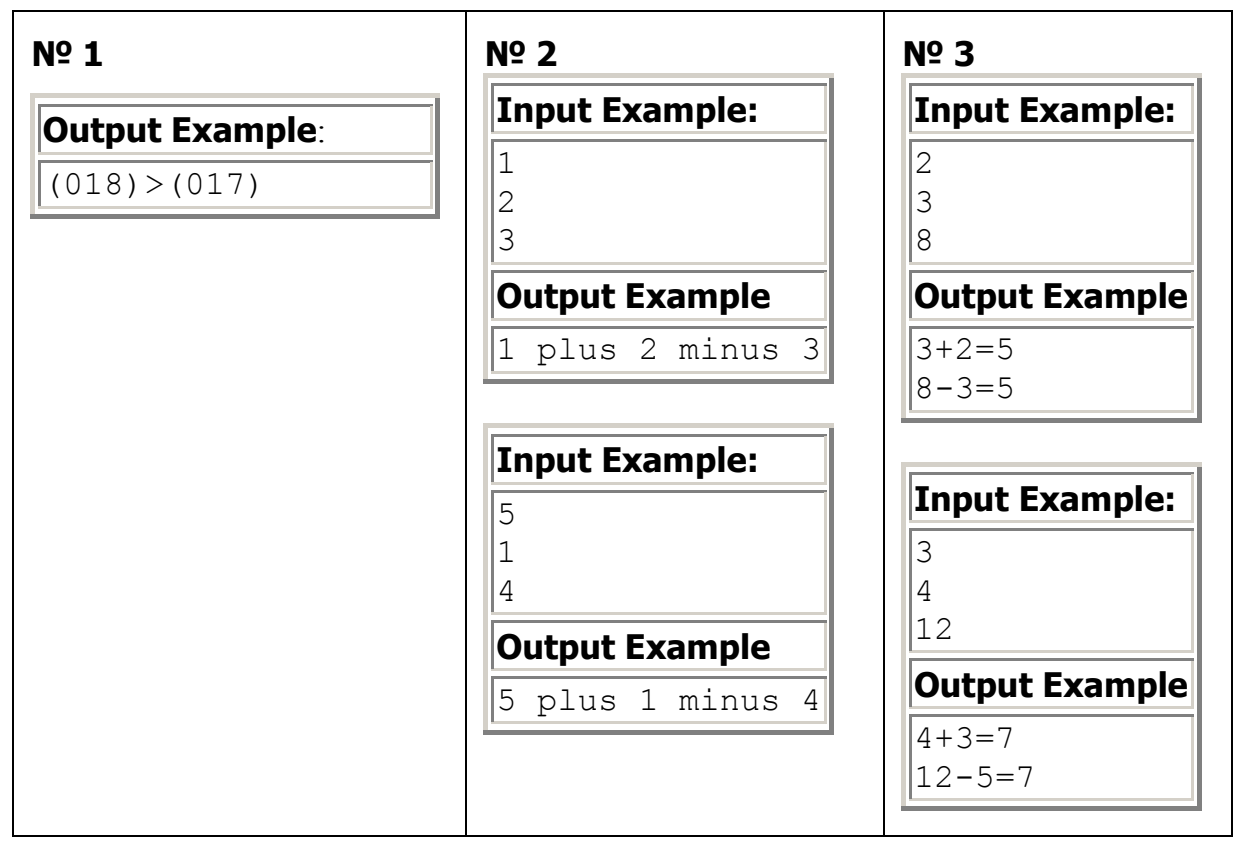

Fig.1. Conditions of Tasks 1-3. 


\section{Developing Interesting Differentiated Training}

The learning process is structured in such a way that, first of all, it is focused on the general development of the child: thinking, memory, independence, attention, hard work and creativity. Practice shows that far from all children continue study programming in secondary school, but certainly everyone gets the development. And the more they do the more development they get.

Since the child is engaged with us until he shows interest, we try to build the learning process as motivated as possible. This is done, first of all, due to the variety of presentation forms and ways of completing tasks. Another area is ensuring assignment of tasks for all pupils.

However, the feasibility of tasks for everyone with a linear system of building tasks leads to the loss of interest in classes for stronger children. That is why we have introduced differentiated training. In each topic, the main tasks are highlighted. They form the backbone of learning. To complete the package of tasks it is enough to complete only them. The strongest children can do so. However, if some main task is performed incorrectly, the pupil is automatically sent to the first facilitating task. Also he can get there if he presses the "I don't know" button. There can be several such assignments, gradually explaining how to complete the main task. Each of the facilitating tasks may have its own system of facilitating tasks, etc. Such a tree of teaching tasks provides a differentiated and adaptive approach, where each pupil can have his own educational trajectory, taking into account not only his general level of training, but also the current psychophysical state during a particular lesson. You're worse in thinking - you often ask for help, move more slowly on educational material. You think well - you ask for help less often, you move faster on educational material.

\section{Task - Oriented Training}

The training material is a set of tasks for the development of programs. If the child cannot solve the problem, he is offered a selection of tasks for the development of programs that gradually lead to the solution of this problem. If a new theory is required to solve the problem, it is given in the most concise and understandable form. If this is not enough, a lot of training tasks are offered for entering answers according to the initial data, compiling algorithms by permuting lines, composing permuting lines of programs according to given algorithms, a set of programs by algorithms, and many others.

\section{Minimalist Approach to Theory}

Theoretical information (Dolinsky, 2013) is given exactly in the quantity that is necessary for solving current problems.

To solve problems 4-10 of the Olympiad in programming in grades $1-4$, you need to know the char, string data types, the concept of how to access the string character $\mathrm{s}$ at 
position i (s [i]), as well as the built-in string processing functions length, copy, delete , pos. These data are entered by us in the learning process.

To solve the problems $11-15$ of the Olympiad in programming in grades $1-4$, you need to know the concept of a one-dimensional array, as well as the following standard algorithms for cyclic processing of one-dimensional arrays: summation of elements; counting elements with a given property (including complex conditions using AND / OR unions); determination of the maximum / minimum element and its number (all use the FOR loop); search for elements that have the specified property (using the WHILE loop operator).

To solve problem 16, one needs to be familiar with the concept of a two-dimensional array and its components (rows, columns, main and secondary diagonals) and be able to apply standard algorithms studied for one-dimensional arrays on a two-dimensional array and its components.

To solve problem 17, you must be able to find the distances: between two points; from one point to several; adjacent distances; all distances between two sets of points. And then apply the studied standard algorithms on one-dimensional and two-dimensional arrays.

To solve problem 18, you need to be able to come up with algorithms for processing strings of characters, practically without learning a new theory, with the exception of several new built-in procedures and functions (str, val, chr, ord, insert).

To solve problem 19, it is required to study and be able to apply any of the algorithms for sorting the elements of a one-dimensional array, for example, "bubble" or "exchange".

To solve problem 20, you need to be able to read a page of text - the conditions of the task and understand what exactly needs to be done (as a rule, apply one of the methods for standard processing of one-dimensional or two-dimensional arrays).

\section{Regional Programming Olympiads for Pupils in Grades 1-4}

It is very important that in the Gomel region 5 regional competitions are held for pupils of grades 1-4, which are held in October (school Olympiad), November (city Olympiad), March (school Olympiad) and April (two: city Olympiad and regional Olympiad). All of them have a standard format of 20 of the above tasks with a slight increase in complexity from the first to the fifth Olympiad. Since all competitions are held on the basis of the DL.GSU.BY website, anyone can take part in each of them. The official results at city and regional Olympiads include, of course, only official participants who decided the Olympiad under the control of the jury.

Training is organized in the direction of the Olympiads (or vice versa, the Olympiads correspond to the learning process). One way or another, the results of the Olympiads accurately show for each pupil which topics he has studied and which topics he has yet to study (or which topics he has learned poorly). And therefore, the Olympiads provide a strong motivating effect on pupils and teachers. 


\section{Preparation for the Olympiads of Grades 5-8}

From the 2016-2017 academic year, the tasks 16-20 of the Olympiad for pupils in grades $1-4$ are exactly the same as the tasks 3-7 of the Olympiad for pupils in grades 5-8. In addition, task 1 of the 5-8 grade Olympiad is a task on the topic "Introduction to Programming" and requires no more skills than the third task (processing the entered numbers and formatted output) of the 1-4 grade Olympiad. And the task 2 of the Olympiad of grades 5-8 is a problem on the topic of a one-dimensional array and, again, it does not require additional knowledge to solve it compared to the tasks of the 11-15 Olympiad for grades 1-4. Thus, it turns out that the best pupils of grades 1-4 can simultaneously participate in the Olympiad of grades 5-8 and not only participate, but also win diplomas in them, since in the Olympiad of 5-8 grades there are 10 problems, seven of which the best pupils can solve Grades 1-4. Thus, it turns out that students in grades $1-4$, preparing for their olympiads, are simultaneously preparing for the olympiads that are waiting for them after moving to the 5 th grade.

\section{Problems in School Mathematics, Olympiad Mathematics and Informatics Mathematics}

On the DL.GSU.BY website there is a course "Mathematics", which contains tasks in mathematics in the program of grades 1-5, Olympiads in mathematics (Kangaroo; Beaver; Canadian, United Kingdom and Texas University Math Contests), informative math. Informatics math problems were obtained by automatically converting programming tasks into math problems, where according to the problem condition, for each of the proposed input data sets; the corresponding result must be manually calculated.

\section{Continuing Education Motivation Contests}

Obviously, the more time a pupil devotes to learning, the faster he will move on the training material, and the better his results at the Olympics. This is especially true in the case of an effective system of automatic differential learning, designed by us. In order to intensify the independent work of pupils at home, with his grandmother, in a sanatorium, etc. we hold Cups: "Autumn", "Winter", "Spring”, "Summer", "Person of the Year". The main goal of this kind of competition is to determine who has solved the more problems for the fall, winter, spring, summer, for the whole academic year (from autumn to summer inclusive), respectively. For pupils in grades 1-4, such competitions are held separately for the courses "Informatics 2015" and "Mathematics". At the time of writing (August, 2019) in the academic year 2018-2019, more than 700 pupils have already taken part in the "Informatics 2015" competition, and more than 100 pupils have taken part in the "Mathematics" competition. 


\section{Many Years of Practical Experience}

The pupils who were the first to study according to the system described above from the 1st grade, finished school in September 2018 (in Belarus it is 11 years of study). It is clear that annual operation and constant feedback led to the improvement in the training system.

Table 1 shows the number of pupils who studied in the competitions "Informatics" and "Mathematics" since the beginning of their conduct.

Table 1

Number of participants in the "Person of the Year" contest

\begin{tabular}{|c|c|c|c|c|c|c|c|c|}
\hline & 2011 & 2012 & 2013 & 2014 & 2015 & 2016 & 2017 & 2018 \\
\hline & $/ 12$ & $/ 13$ & $/ 14$ & $/ 15$ & $/ 16$ & $/ 17$ & $/ 18$ & $/ 19$ \\
\hline Informatics & 249 & 301 & 144 & 166 & 335 & 655 & 760 & 755 \\
\hline Mathematics & - & 146 & 43 & 118 & 131 & 144 & 257 & 135 \\
\hline
\end{tabular}

\section{Experience-based Training Scalability}

On the basis of the DL.GSU.BY website, classes are conducted with primary school pupils by teachers from several educational institutions of Belarus, Russia, Armenia. I want to talk in more details about such work for younger schoolchildren in St. Petersburg. In the summer of 2017, the parent of one of the pupils who studied on our website, created a VKontakte group https://vk.com/spb_dl. The group has information about our system, invite to use DL.GSU.BY, serves for the experience exchange of parents, teachers and pupils.

At the time of writing of the article, there are already 4,150 subscribers. 1065 elementary pupils from St. Petersburg are taking the course "Informatics 2015". Along the way, obviously, according to the information received from this group, elementary school pupils from other cities of Russia began to study in the Informatics 2015 course: Tula, Chelyabinsk, Novokuznetsk, Mytishchi, Vologda, Ozersk.

\section{Productivity}

Children who study in the system described above on the basis of the DL.GSU.BY website regularly become graduates of city, regional, republican and international competitions (http://dl.gsu . by/olymp/result.asp). 


\section{Low Requirements for Teacher Professional Qualifications}

The main advantage of teaching using the DL.GSU.BY website is automatic differentiated learning.

A teacher is required to:

- Open / close class.

- Be friendly to children.

- Maintain a working atmosphere in the classroom.

- Explain children how to work at home.

But, of course, if the teacher is interested in a better result, he himself will want to study, and will begin to think how to motivate / teach better.

\section{"Accelerated Course 2013"}

Initially, the package of tasks "Accelerated Course - 2013" was developed and implemented in the training course "Basic Programming", for pupils of grades 5-8. It contains assignments on 8 topics. The first 8 tasks in computer science Olympiads for pupils in grades 5-8: introduction to programming, one-dimensional array, two-dimensional array, geometry, strings, sorting, text problem, research. Each of the topics includes folders: "Technical minimum", "Olympiad 1-4 grades", "Olympiad 5-8 grades". This ensures the fastest possible advancement in the educational material of the most capable pupils.

In the learning process, it turned out that there are quite a few pupils in elementary school for whom the usual rate of learning slows down their development. Then "Accelerated Course - 2013" was copied from the course "Basic Programming" to the course "Informatics 2015 ". There are currently about 30 such pupils.

\section{Support for the Transition to the Study of $\mathrm{C}++$}

The system of automatic instruction in C++ programming (Dolinsky, 2017), in which all training tasks are generated on the fly based on the author's $\mathrm{C}++$ solutions to the proposed problems, has been introduced on the DL.GSU.BY website since the summer of 2016. Practice has shown that the most natural and simple transition to $\mathrm{C}++$ is carried out after studying the "Accelerated Course -2013", on the initiative and if the pupil wishes.

\section{Conclusion}

A system for teaching text programming in an elementary school, built on the basis of the DL.GSU.BY website at F. Skorina Gomel State University is described in this article. This system has the following advantages: zero entry threshold; propaedeutics 
of text programming; developing, developing, interesting, differentiated training; task oriented training; minimalist approach to theory; regional programming Olympiads for pupils in grades 1-4; preparation for the Olympiads of grades 5-8 (general tasks with the Olympiads of grades 5-8); problems in school mathematics, Olympiad mathematics and informatics mathematics; competitions motivating to permanent studies; many years of practical experience; experience-based training scalability; productivity; low requirements for professional qualifications of teachers; "Accelerated Course 2013" and support for the transition to the $\mathrm{C}++$ study.

\section{References}

Dolinsky M. (2013). An approach to teach introductory-level computer programming. Olympiads in Informatics, 7, 14-22.

Dolinsky M. (2014). Technology for the development of thinking of preschool children and primary school children. Olympiads in Informatics, 8,63-68.

Dolinsky M. (2016). Gomel training school for Olympiads in Informatic. Olympiads in Informatics, 10, 237247.

Dolinsky M. (2017). A New Generation Distance Learning System for Programming and Olympiads in Informatics

Dolinsky M.,Dolinskaya M (2018). How to Start Teaching Programming at Primary School. Olympiads in Informatics, 12, 13-24.

Dolinsky M.,Dolinskaya M (2019). Training In Writing The Simplest Programs From Early Ages, 13, 21-30.

Performance Statistics of Gomel pupils at international and national olympiads in informatics since 1997 up to 2019 (In Russian). http://dl.gsu.by/olymp/result .asp

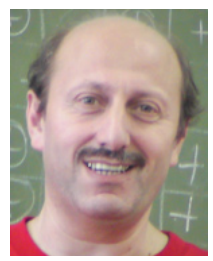

M. Dolinsky is a lecturer in Gomel State University "Fr. Scoryna" from 1993. Since 1999 he is leading developer of the educational site of the University (dl.gsu.by). Since 1997 he is heading preparation of the scholars in Gomel to participate in programming contests and Olympiad in informatics. He was a deputy leader of the team of Belarus for IOI'2006, IOI'2007, IOI'2008 and IOI'2009. His PhD is devoted to the tools for digital system design. His current research is in teaching Computer Science and Mathematics from early age.

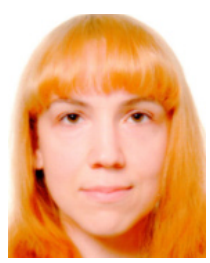

M. Dolinskaya is student in Gomel State University "Fr. Scoryna" from 2005 then graduate student from 2017. Since 2006 she is one of developer of the educational site dl.gsu.by as well as teacher of pupils from first grade. Her current research is in teaching programming from early age. 